\title{
RESPONSABILIDAD CORPORATIVA Y DE LAS PARTES INTERESADAS: VOLVIENDO LA ÉTICA DE NEGOCIOS UNA CONVERSACIÓN DE DOBLE VÍA
}

\author{
Jerry D. Goodstein y ANDrew C. Wicks
}

\section{RESUMEN}

Teniendo en cuenta que las corporaciones se han convertido en las instituciones más poderosas del planeta, icómo sería posible mantener en ellas una actitud ética que incluya las visiones de los proveedores y de los clientes? Se hace necesario iniciar un diálogo constructivo entre las partes interesadas. No se trata de culpabilizar a los actores, sino de lograr un acuerdo sobre lo esencial de sus relaciones. Los autores llaman este importante recurso Responsabilidad corporativa de las partes interesadas. Indican que existen dos formas de pensar acerca del término responsabilidad. En la primera, se entiende que somos responsables "cuando respondemos por nuestra conducta y asumimos nuestras obligaciones". En el segundo, es necesario volver a la raíz latina del término, respondere que significa responder o comprometerse de nuevo. Para los autores, la palabra incluye los dos sentidos. Las partes interesadas no sólo son los receptores de las acciones organizacionales, sino también actores que deben considerar cómo sus acciones pueden dañar a las compañías y a las otras partes interesadas.

La responsabilidad de las partes interesadas incluye varias ventajas: reorienta la forma de pensar sobre la ética de los negocios; colabora en la explicación de fallas morales en las organizaciones; ayuda a diseñar corporaciones donde los desastres o fallas son raros; favorece el éxito basado en la excelencia y el desempeño correcto, y desarrolla los conceptos que facilitan el desarrollo de nuevos negocios dentro del ámbito del beneficio general.

\section{PALABRAS CLAVE}

Responsabilidad, ética en los negocios, clientes, provee- dores, partes interesadas, diálogo, desempeño sobresaliente.

\section{ABSTRACT}

Taking into account the fact that corporations have become the most powerful institutions on the planet, would it be possible for them to maintain an ethical attitude, including the visions of suppliers and clients?

It is necessary to start a constructive dialog between stakeholders. It's not about blaming the actors, but rather about reaching an agreement on what is essential in their relationships. The authors call this important resource Stakeholder corporate responsibility.

They indicate there are two ways of thinking about the term, responsibility. In the first one, it is understood that we are responsible "when we answer for our conduct and are accountable for our obligations". In the second, it is necessary to come back to the Latin root of the term, respondere which means to answer or commit anew. For the authors, the word includes the two senses of the word. Stakeholders are not only receivers of organizational actions, but also actors who should consider how their actions may harm companies and other interested parties.

Stakeholder responsibility includes a number of advantages: it refocuses thinking on business ethics; it helps explain moral failings in organizations; it helps to design corporations where disasters or failures are rare; it favors success based on excellence and proper performance, and it develops concepts which facilitate the development of new businesses within the scope of the general good. 
Las corporaciones se han convertido en las instituciones más poderosas sobre el planeta, de tal forma que tiene sentido hablar acerca de las responsabilidades de sus líderes con las partes interesadas. Pero, ¿qué pasa si invertimos esta conversación? En lugar de suponer que la ética de negocios sólo trata acerca de cómo se comportan las corporaciones, ¿qué pasa si consideramos el papel de las partes interesadas (empleados, clientes, inversionistas, proveedores, miembros de la comunidad) en desarrollar prácticas de negocios éticas y responsables en lo ambiental?

Pensamos que es hora de comenzar un diálogo a cerca de la responsabilidad de las partes interesadas. Esta es la razón por la que nos llamó la atención oír de una compañía tabacalera que estaba tratando de involucrar a las partes interesadas en un esfuerzo conjunto para enfrentarse a los temas económicos, sociales, ambientales, y de salud asociados con el producto de la compañía. Y a los ejecutivos de la compañía les llamó la atención oír a cerca de nuestro trabajo y sobre la idea de la responsabilidad de las partes interesadas -tanto les llamó la atención que nos dieron pasajes para que fuéramos a conocerlos-. Resultó que nuestros intereses no se traslapaban; ellos vieron en el lenguaje de la responsabilidad de partes interesadas una forma de quitarse

\section{RESEÑA AUTOR:}

Jerry D. Goodstein es un Fellow Batten y profesor de administración y operaciones en la Universidad Estatal de Washington, Vancouver. Él puede ser contactado en goodstei@vancouver.wsu.edu.

Andrew C. Wicks es profesor asociado de administración de empresas en la Escuela Darden y codirector del Centro Olsson para la Ética Aplicada. Él puede ser contactado en WicksA@darden.virginia.edu. de encima la sanción del público y avergonzar a las partes interesadas, en este caso las organizaciones no gubernamentales, para que actuasen de ciertas formas.

Jugar a culpar a las partes interesadas no está en nuestra agenda, ni queremos dirigir la atención hacia ellas porque creemos que la sociedad ha sido demasiado dura con las corporaciones. Para nosotros, la responsabilidad de las partes interesadas es una forma de pensar acerca de volver la ética de negocios asunto de todos.

Hay dos maneras de pensar acerca del concepto de "responsabilidad". En un sentido, somos responsables cuando respondemos por nuestra conducta y asumimos nuestras obligaciones. El otro sentido de la palabra proviene de sus raíces latinas ("respondere"), que significa responder, o "comprometerse de vuelta". Nuestra definición de responsabilidad incluye ambos significados y por tanto se extiende a la definición tradicional de partes interesadas como individuos, grupos u organizaciones potencialmente afectadas por las acciones y políticas de una organización. Ciertamente, creemos que las partes interesadas no sólo son los receptores de las acciones organizacionales, sino actores también, quienes deben considerar cómo sus acciones pueden dañar a las compañías como también a otras partes interesadas.

Cuando las partes interesadas descuidan sus responsabilidades, los costos pueden ser abrumadores. En 2002, el robo y fraude por parte de empleados se estimó en $\$ 600$ mil millones, aproximadamente 6\% del PBI. Aunque el crimen de alto nivel da cuenta de una gran proporción de dicho comportamiento corrupto, aún está ocurriendo una cantidad considerable de robo y fraude 
organizacionales más bajos, tales como el uso doméstico de suministros, la manipulación de cuentas de gastos, y fraude con cheques. Y el contexto ético de los negocios se corrompe aún más por consumidores que se dedican al robo de televisión por cable, fraude con seguros de autos, y abuso de políticas de devolución por parte de clientes, para mencionar solo unos pocos ejemplos.

Antes de comentar por qué importa la responsabilidad de partes interesadas, puede ser útil destacar un par de ejemplos. Considérese, en primer término, Enron (podríamos, por supuesto, señalar muchas otras corporaciones), donde la alta dirección se involucró en un fraude masivo por un monto de varios miles de millones de dólares. Los empleados de más bajo nivel, siguiendo el liderazgo de la alta dirección, manipularon los precios de la energía en California a sabiendas y hacían chistes al respecto con sus pares. Salirse con este fraude por tanto tiempo requirió la participación activa y pasiva de muchas partes interesadas - auditores que miraban al techo y daban el visto bueno a los libros-; una junta directiva que no hizo suficientes preguntas y dejó las reglas éticas de lado dos veces para los altos ejecutivos; bancos de inversión que estaban dispuestos a facilitar transacciones corruptas para poder ganarse el favor de la firma; y los accionistas que no preguntaron por qué los reportes de retornos accionarios positivos parecían demasiado buenos para ser ciertos.

En contraste, la muerte de Enron con eBay, una compañía que no solo sobrevivió el estallido de la burbuja de internet sino que ha prosperado. Debido a que la existencia de eBay depende de su habilidad para sostener una comunidad de partes interesadas en línea -empleados, compradores, y vendedores- la compañía ha trabajado duro para desarrollar prácticas que refuercen la responsabilidad mutua, tales como carteleras donde los usuarios se apoyan unos a otros, salas de chat, y boletines de noticias. Una característica particularmente importante de la comunidad eBay es el Feedback Forum (Foro de Retroalimentación), donde los compradores y vendedores se evalúan unos a otros. Este tipo de realimentación resulta en un perfil de usuario, que sigue a los compradores y vendedores por todas partes en $e B a y$, engendrando así la responsabilidad y confianza mutua dentro de la comunidad.

Por supuesto que Enron y eBay no son ni villanos ni santos, pero sus interacciones con las partes interesadas apuntan hacia lo que vemos como crucial y prometedor acerca de la responsabilidad de las partes interesadas y las preguntas que presenta tanto para investigadores como para administradores.

\section{¿POR QUÉ IMPORTA LA RESPONSABILIDAD DE LAS PARTES INTERESADAS? \\ 1. REORIENTA LA FORMA COMO PENSAMOSY YABLAMOS ACERCA DE LAÉTICA DE NEGOCIOS.}

Los académicos y practicantes han usado el lenguaje de la responsabilidad corporativa para motivar a las firmas a hacer de la ética una práctica integral. El lenguaje de la responsabilidad de las partes interesadas es otra manera de entretejer la ética con las interacciones de los negocios. También provee un marco teórico lo suficientemente amplio como para abarcar todo lo que sucede dentro y alrededor de los negocios, destacando la
Los académicos ypracticantes han usado el lenguaje de la responsabilidad corporativa para motivar a las firmas a hacer de la ética una práctica integral. El lenguaje de la responsabilidad de las partes interesadas es otra manera de entretejer la ética con las interacciones de los negocios. 
idea de que todas las partes están motivadas, como también constreñidas, por deberes morales. Aunque la responsabilidad de las partes interesadas se traslapa con varias teorías, tales como los comportamientos de ciudadanía organizacional, responsabilidad social corporativa, ética, y teoría de las partes interesadas, tiene un foco específico que está ausente en gran medida de nuestras conversaciones y de la bibliografía existente: nos recuerda -como administradores y académicos- que necesitamos gastar tanto o más tiempo preocupándonos por lo que las partes interesadas están haciendo, y por qué lo gastado, respecto de lo que hacen las corporaciones.

La responsabilidad de las partes interesadas también le da a los ejecutivos de las empresas una poderosa herramienta retórica para involucrar a las partes interesadas y engendrar soluciones cooperativas y prácticas a los problemas. Los ejecutivos tabacaleros con los que hablamos reconocieron de inmediato el potencial de esta herramienta. Desafortunadamente, ellos pasaron de involucrar a forzar. Pero era un comienzo.

Finalmente, la responsabilidad de las partes interesadas ayuda a mantener una tensión productiva entre los intereses teóricos de los dedicados a la ética empresarial y la orientación práctica de los administradores. Provee un punto de referencia para la excelencia como también para la conducta despreciable en un entorno práctico, pero también se enfoca en el comportamiento y acciones concretas. La responsabilidad de las partes interesadas nos mueve a pensar acerca de la teoría y la práctica en forma simultánea, en lugar de hacer una teoría, bien sea de los negocios o de la ética, y luego aplicarla a la otra área.

\section{AYUDA AXPLICAR LAS FALLAS MORALES EN LAS CORPORACIONES, ASIGNANDO CULPA A DIVERSAS PARTES INTERESADAS.}

Pese a lo que la alta dirección y los medios tratan de sugerir, todos sabemos que la mayoría de los escándalos son el resultado de las acciones de una amplia gama de personas -no sólo unas pocas manzanas podridas-. Esta no es una idea nueva, sino que la responsabilidad de las partes interesadas nos recuerda que al pensar acerca de por qué ocurren las rupturas éticas, necesitamos comenzar mirando las interacciones entre participantes clave. Las compañías (y las comunidades) trabajan bien debido a valores y acuerdos compartidos. Especialmente en las organizaciones, los individuos desarrollan rutinas, prácticas, y una comprensión de responsabilidades que crean contrapesos, como errores, abusos, y otras formas de oportunismo. Llamamos estas normas compartidas, acuerdos, y prácticas "regímenes de responsabilidad", maneras formales e informales en las que los individuos trabajan juntos para hacer las cosas y evitar las rupturas éticas.

La falla organizacional puede ser una oportunidad para mirar de cerca los regímenes de responsabilidad para poder identificar dónde ocurrieron las rupturas y como contribuyó cada parte interesada al lío. Es claro que los eventos en Enron fueron el resultado de lapsos masivos en los regímenes de responsabilidad. El cuadro es complicado. Pero gastar tiempo dilucidándolo será crítico si la comunidad empresarial en general desea diagnosticar la enfermedad y comenzar a pensar en una cura, en lugar de simplemente sacar unos pocos 
chivos expiatorios por la puerta, generando buenas relaciones públicas, volviendo al trabajo, y esperando que no vuelvan a pasar cosas malas.

\section{NOS AYUDA P PENSAR EN CÓMO CREAR ORGANIZACIONES EN LAS QUE LOS DESASTRESY FALLASSON RARAS.}

Si nos tomamos el tiempo para entender los sistemas interconectados de responsabilidad de las partes interesadas que pueden prevenir, detectar y enfrentar las rupturas éticas, estaremos en mucho mejor posición para arreglarlos cuando fallen. La ley y los incentivos de mercado ciertamente son herramientas poderosas para alinear los intereses de las partes interesadas con sus responsabilidades legales y morales, pero son costosos, imperfectos, y pueden enviar señales potencialmente disfuncionales. Mucho del debate reciente en los medios acerca de los escándalos corporativos se ha enfocado en cambiar el sistema de gobierno corporativo, examinar la remuneración de ejecutivos, mejoramiento de la composición de la junta y relaciones con la dirección, y buscar legislación para hacer más responsables a los ejecutivos y juntas corporativas. Pensamos que esta es una conversación que vale la pena, pero desconoce la realidad de que el comportamiento responsable en las corporaciones depende de una amplia gama de interacciones interpersonales.

Considérense los empleados. Una cultura corporativa ética depende de empleados que tienen el valor de hablar cuando son testigos de actos irresponsables, incluso frente a la resistencia de sus superiores. Pero en realidad, la gente que sí lanza la alerta, tales como Cynthia Cooper de WorldCom y Sherron Watkins de Enron, son héroes en parte por lo escasos que son. Si las únicas opciones de los empleados son salir de la firma o exhibir lealtad ciega, seguirán existiendo problemas grandes con la corrupción corporativa. Mejorar el desempeño de las compañías (y partes interesadas) requiere que los ejecutivos piensen acerca de la reconstrucción de las organizaciones desde abajo y de afuera hacia adentro, como también de arriba hacia abajo, y crear regímenes de responsabilidad que son robustos y perdurables.

\section{NOS AYUDA A PENSAR ACERCA DE CÓMO CREAR ORGANIZACIONES DESTACADAS POR LA EXCELENCIA YDESEMPEÑO SOBRESALIENTE.}

La responsabilidad de las partes interesadas ya está implícita en la forma como muchas compañías piensan acerca de y logran el desempeño sobresaliente. Crecientemente, las compañías tienen que ganarse las mentes y corazones de partes interesadas clave para mantener sus mejores empleados, generar productos de alta calidad, mantener lealtad del cliente, y construir relaciones eficientes y productivas con proveedores. La premisa es que las partes interesadas deben ir más allá de cumplir con los deberes contractuales o responder a incentivos específicos. Ellos necesitan sentir el compromiso y lealtad para con la misión y valores de la compañía.

Esto es precisamente lo que es poderoso respecto de eBay. Esta comunidad depende de sus miembros para proteger el sitio y unos a otros. Haciendo uso de la lealtad de los usuarios para con la comunidad de eBay, la compañía ha desarrollado un sistema de auto regulación que, junto con otras formas de gobierno, permite que un conjunto de usuarios muy diversos y dispersos compren y vendan casi cualquier cosa por la inter-
La leyylos incentivos de mercado ciertamente son herramientas poderosas para alinear los intereses de las partes interesadas con sus responsabilidades legales $y$ morales, pero son costosos, imperfectos, $y$ pueden enviar señales potencialmente disfuncionales. 
Hewlett-

Packardy Office Depot tenían poca experiencia en manejar los desperdicios electrónicos, pero ambas compañías reconocieron

la tensión entre suénfasis en la gestión ambiental y la proliferación de sus productos en rellenos sanitarios. net. Esta actividad cooperativa refuerza un sentido de conexión a propósitos y valores compartidos. La creación de una organización tal como eBay donde la gente se quiere volver parte interesada -es decir, asumir las responsabilidades de estar afiliado con la firma- es integral a la creación de un negocio sobresaliente.

\section{NOS DA UN LENGUAJE PARA CONCEPTUALIZARY YRABAJAREN RETOS DE NEGOCIOS NUEVOS.}

Las exigencias sobre las corporaciones están aumentando, como también las expectativas por su habilidad para responder al activismo de partes interesadas y otros tipos de retos de negocios. Una cantidad de compañías han usado la idea de responsabilidad de partes interesadas -explicita o implícita- para idear soluciones cooperativas a tales retos.

Por ejemplo, Hewlett-Packard y Office Depot tenían poca experiencia en manejar los desperdicios electrónicos, pero ambas compañías reconocieron la tensión entre su énfasis en la gestión ambiental y la proliferación de sus productos en rellenos sanitarios. Ellos también reconocieron que ninguna organización o grupo de partes interesadas por sí sólo podía acometer un programa así de masivo solo. De manera que vincularon a una gama de partes interesadas en un experimento: a lo largo de un período de ocho semanas desde Julio hasta Septiembre de 2004, Office Depot abrió sus 901 almacenes para entregas, por parte de clientes, de equipo electrónico viejo (no necesariamente productos HP). Durante este periodo, los clientes dejaron más de 325.000 productos con un peso de más de 10,5 millones de libras, tales como monitores, PCs, impresoras, escaners, teléfonos celulares, y televisores. Office Depot y HP compartieron los costos de despachar estos productos a facilidades de HP en California y Tennessee.

La reciente Iniciativa Rethink (Repensar) coordinada a través de eBay avanza sobre dichos esfuerzos y junta a la industria (Apple, Dell, HP, IBM, Intel), gobierno (EPA), y organizaciones ambientales (Coalición de Tóxicos del Valle de la Silicona) en un esfuerzo conjunto para enfrentar el reto de los desperdicios electrónicos. Como parte de este esfuerzo, eBay está suministrando información acerca de las opciones privadas, públicas, y sin ánimo de lucro en su sitio Web.

Lo claro en estos ejemplos es la necesidad de colaboración. Los investigadores que estudian las colaboraciones medioambientales entre compañías y partes interesadas tales como aquellas entre la Alianza por la Innovación Ambiental y socios corporativos tales como UPS, Norm Thompson Outfitters, y SC Johnson, han encontrado una cantidad de responsabilidades que son críticas para el éxito de estas sociedades: crear valor mutuo a través de perspectivas y conocimiento compartidos, establecer legitimidad para los esfuerzos cooperativos con partes internas y externas, y construir confianza entre socios de firmas y partes interesadas.

\section{UNA AGENDA DE LAS PARTES INTERESADAS}

Parte de lo que ha sido gratificante en nuestras conversaciones con compañías tales como Starbucks, Hewlett-Packard, y Office Depot es lo implícita que está la idea de las partes interesadas. Aunque muchos no utilizaron el término, los ejecutivos con los que hablamos estaban buscando 
involucrar una amplia gama de partes interesadas.

Esperamos que nuestro trabajo sugiera una agenda tanto para los practicantes como para los académicos. Los líderes pueden comenzar por hacer preguntas básicas relacionadas con sus propias partes interesadas: ¿Qué significa ser un cliente responsable? ¿Un proveedor responsable? ¿Un inversionista responsable? Aunque alentamos tanto a administradores como a los investigadores a que hagan estas preguntas amplias, también queremos que consideren respuestas a estas más individuales y específicas a la organización. Es decir, el papel que una cierta parte interesada (un empleado, por ejemplo) juega para Starbucks puede ser muy distinto al papel que él o ella juega para Wal-Mart, y los tipos de responsabilidad que el empleado puede tener que asumir también variará a lo largo de los contextos organizacionales.

Citigroup dio un paso notable al pedir a sus 260.000 empleados que reflexionaran acerca de la historia de Citigroup y su responsabilidad por construir sobre su legado a través de responsabilidades compartidas de unos con otros, clientes, y con la compañía. Otras compañías tales como J\&J, Southwest Airlines, y Xerox hacen énfasis similar en la responsabilidad de los empleados, mientras las compañías tales como Intel y HP destacan la importancia de la responsabilidad de los empleados en cuanto a defender los valores de la organización y proteger su marca.

Una vez que los administradores han confrontado las preguntas básicas y han determinado las responsabilidades relevantes, necesitarán traducir esas responsabilidades en expectativas de las partes interesadas y comunicarlas de manera clara. De la misma forma que las partes interesadas han hecho de sus expectativas un cimiento crítico para la responsabilidad corporativa, las firmas tienen que comunicar sus expectativas respecto del comportamiento responsable a las partes interesadas. Puede haber una variedad de maneras para que los administradores premien a las partes interesadas, tales como dar incentivos para que los clientes reciclen productos, renovando contratos con proveedores ejemplares, y haciendo que la caracterización por parte del empleado de los valores de la firma, sea componente de la evaluación de desempeño y de las recompensas.

A medida que las firmas construyen relaciones más cercanas con menos partes interesadas, y a medida que la presión pública a favor de mayor responsabilidad corporativa se intensifica, las firmas deben depender aún más de las partes interesadas para ayudar a que la organización logre sus metas y defienda sus valores centrales.

La agenda de investigación que surge del concepto de la responsabilidad de las partes interesadas hace un llamado para que los dedicados a la ética empresarial, administradores, y científicos sociales trabajen juntos y se comuniquen con audiencias por fuera de las fronteras académicas y de las firmas tales como clientes, proveedores, e inversionistas. El lente de la responsabilidad de las partes interesadas motiva la investigación empírica de la variedad de fuerzas que conforman el comportamiento individual y de grupo dentro de las organizaciones y que influencian en los regímenes de responsabilidad. Los negocios se van incrementando a través de redes y alianzas de firmas y partes interesadas. Los investigadores pueden ver cómo operan dichas redes para entender 
Miles de millones de dólares se han perdido porque los auditores, juntas directivas decorporaciones, bancos de inversión, y accionistas perdieron de vistasus responsabilidades fundamentales. mejor cuáles condiciones tienden a hacer que los diversos actores en un grupo de partes interesadas abracen y defiendan las responsabilidades clave y qué condiciones tienden a hacer que ellos rechacen y eviten esas responsabilidades. Los investigadores también necesitan examinar qué pasa cuando las firmas y las partes interesadas cumplen sus mutuas responsabilidades. Por ejemplo, las partes interesadas pueden convertirse en socios poderosos en el desarrollo de innovaciones en productos y servicios y en mejorar iniciativas de sostenibilidad. Los investigadores también pueden usar la responsabilidad de las partes interesadas como un lente para pensar acerca de la naturaleza de las organizaciones y qué hace que tengan éxito o fallen.

A medida que los académicos y los practicantes trabajan juntos para entender las dinámicas de la vida en las organizaciones desde el punto de vista clave de las partes interesadas clave, en particular en cómo las partes interesadas se distancian de la res- ponsabilidad, cómo los regímenes de responsabilidad se rompen, en la forma como podemos hacer puntos de referencia y diseñar sistemas de responsabilidad más fuertes, la sociedad estará en una mejor posición para combatir la corrupción empresarial y alentar la sostenibilidad. Sin alguna noción de la responsabilidad de partes interesadas, los negocios no funcionan. Miles de millones de dólares se han perdido porque los auditores, juntas directivas de corporaciones, bancos de inversión, y accionistas perdieron de vista sus responsabilidades fundamentales. ¿Cuánto daño adicional será hecho si las firmas y las partes interesadas fallan en reconocer y cumplir sus responsabilidades de proteger el medio ambiente? Desarrollando ideas robustas acerca de la responsabilidad de las partes interesadas y sistemas eficientes e inteligentes que refuercen tales ideas, los líderes de las empresas estarán tanto más cerca de crear compañías excelentes y mercados excelentes. Al final, la ética se volverá asunto de todos.
Batten Briefing, Volumen 5, Number 1. Translater and published with permission by the Batten Intitute at the Darden Graduate School of Business Administration, University of Virgina.
Batten Briefing, Volumen 5, Número 1. Traducido y publicado con el permiso del Instituto Batten en el Darden Graduate School of Business Administration, Universdad de Virginia. 